\title{
Protein-Mediated Nucleation of Nanoparticles In-Situ
}

Sanjay Kashyap ${ }^{1}$, Taylor Woehl ${ }^{1}$, Miguel S. Sanchez-Quesada ${ }^{2}$, Carmen Valverde-Tercedor ${ }^{2,3}$, Concepcion Jimenez-Lopez ${ }^{2}$, Surya K. Mallapragada ${ }^{1,4}$, and Tanya Prozorov ${ }^{1}$

${ }^{1}$ Division of Materials Sciences and Engineering, US DOE Ames Laboratory, Ames, IA, 50011, USA.

${ }^{2}$ Department of Microbiology, University of Granada, Spain.

${ }^{3}$ Max Plank Institute for Colloids and Interfaces, Potsdam, Germany.

${ }^{4}$ Chemical and Biological Engineering, Iowa State University, Ames, IA, 50011, USA.

Magnetic nanoparticles with narrow size distribution and large magnetic moment per particle are in high demand in various technologically important areas, from data storage and quantum computing, to magnetocaloric refrigeration and cancer therapy. For most applications, uniform size, controlled shape and well-defined magnetic properties are essential [1].

In living organisms, nucleation and crystallization of biogenic inorganic materials is controlled by diverse biomineralization proteins. Inspired by Nature, biomimetics allows low-temperature fabrication of new magnetic functional nanostructures, using synthetic polymers, viruses, peptides, DNA molecules, proteins and various polymer-based hybrid materials as matrices, scaffolds and templating agents [2-4]. However, the nature of control the protein exerts over the nucleation and growth of a certain mineral phase, its size and shape, is not well understood, because the mechanism a biomimetic process is usually deduced from the post-synthesis analysis of a specimen. While this approach yields important information about the properties of a resulting material, it does not permit dynamic characterization of the biomimetic processes.

Recent advances in in-situ Scanning Transmission Electron Microscopy with a fluid cell allow characterization of the protein self-assembly and specific binding in a fully hydrated state [5-7]. We report here on the in-situ nucleation of nanoparticles mediated by two acidic bacterial recombinant proteins, Mms6 and MamC. We have visualized micelles of Mms6 and MamC recombinant ironbinding proteins in-situ by utilizing the STEM-HAADF contrast enhancement of surface bound iron species. During the in-situ reaction, nanoparticles form preferentially on the surface of protein micelles, pointing to the importance of presence of extended protein surface in their formation.

Our results represent a significant step forward in understanding the process of self-organization of the recombinant proteins employed in biomimetic synthesis, specific metal binding of proteins, and their role in the templated nanoparticle nucleation. Our findings are applicable for the in-situ characterization of a variety of inorganic-organic interfaces in protein solutions. 


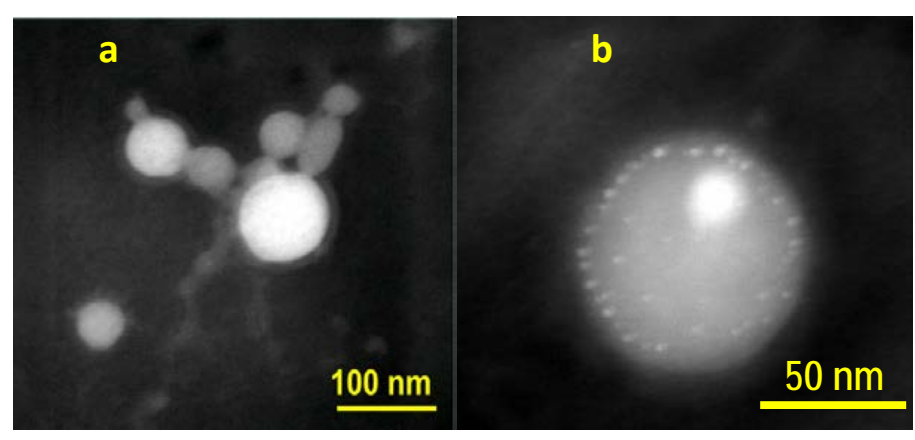

Figure 1. STEM HAADF image of iron-incubated proteinateous micelles on the functionalized silicon nitride window membrane taken in a fluid flow mode: (a) diffusion of iron into the surrounding liquid results in a "halo"effect; (b) iron oxide nanoparticls form in-situ on the surface of the Mms6- micells after delivering the dilute $\mathrm{NaOH}$ solution to the fluid cell.

\section{References}

1. Thanh, N.T.K., Magnetic Nanoparticles: From Fabrication to Clinical Applications. 2012, India: CRC Press Taylor\&Francis Group.

2. Addadi, L., S. Weiner, and M. Geva, On how proteins interact with crystals and their effect on crystal formation. Z Kardiol 2001. 90(Suppl 3): p. 92-98.

3. Klem, M.T., M. Young, and T. Douglas, Biomimetic magnetic nanoparticles. Materials Today, 2005. 8(9): p. 28-37.

4. Prozorov, T., et al., Novel Magnetic Nanomaterials Inspired by Magnetotactic Bacteria: Topical Review. Materials Sciences and Engineering R: Reports 2013. 74(5): p. 133-172.

5. Klein, K.L., I.M. Anderson, and N. De Jonge, Transmission electron microscopy with a liquid flow cell. Journal of Microscopy, 2011. 242(2): p. 117-123.

6. Murray, C.B., Watching nanocrystals grow. Science (Washington, DC, United States), 2009. 324(5932): p. 1276-1277.

7. Ring, E.A. and N. de Jonge, Microfluidic System for Transmission Electron Microscopy. Microscopy and Microanalysis, 2010. 16(05): p. 622-629. 